\title{
Editorial
}

Z Gerontol Geriat 2012 · 45:448-449

DOI 10.1007/s00391-012-0364-9

Online publiziert: 19. Juli 2012

(c) Springer-Verlag 2012

\section{Gosch ${ }^{1} \cdot$ K. Pils ${ }^{2}$}

${ }^{1}$ Landeskrankenhaus Hochzirl, Zirl

${ }^{2}$ Institut für Physikalische Medizin und Rehabilitation, Sozialmedizinisches Zentrum Sophienhospital, Wien

\section{Polypharmazie im Fokus der Geriatrie}

die Deutsche Gesellschaft für Geriatrie [6] als auch die Österreichische Gesellschaft für Geriatrie und Gerontologie [7] haben dieser Entwicklung Rechnung getragen und Arbeitsgruppen zum Thema Polypharmazie eingerichtet. Beide Arbeitsgruppen haben wesentlich dazu beigetragen, dass dieses Thema in den Fokus der geriatrischen Medizin gerückt ist. Wie - Abb. 1 zeigt, haben die PubMed-Publikationen zur Polypharmazie seit dem Jahr 2000 im Vergleich zu Frailty deutlich zugenommen. Die relative Zuwachsrate erreicht zwar nicht das Ausmaß der Publikationen zu Frailty, allerdings bestätigt der kontinuierliche Anstieg das wachsende Interesse an der Thematik.

Die Anzahl der verordneten Medikamente ist der stärkste Prädiktor für das
Auftreten einer ADR [8]. Zahlreiche Initiativen und Tools sind Negativlisten und zielen auf eine Reduktion der Substanzen ab. Zusätzlich werden die Listen im Hinblick auf potenziell inadäquate Substanzen für ältere Patienten fortlaufend überarbeitet und auch für mehrere Länder an die eigenen Gegebenheiten angepasst [9, 10]. Im Mittelpunkt steht somit das Management der Polypharmazie. Trotz aller vielversprechenden Ansätze fehlt nach wie vor die Evidenz, dass die Anwendung derartiger Listen zu einem besseren Outcome für die Patienten führt [11]. Als Paradoxon der geriatrischen Pharmakotherapie kann man den Zusammenhang zwischen Polypharmazie und medikamentöser Unterversorgung, dem „undertreatment“, bezeichnen [12]. Gerade dieser Zusammen-
Im Mittelpunkt des Interesses steht zunehmend die Patientensicherheit. Die Risiken von Nebenwirkungen und Interaktionen, sowohl von „drug-drug interactions“ als auch von "drug-disease interactions", sind kaum mehr überschaubar. In einer von Lazarou et al. [4] durchgeführten Metaanalyse wurde geschätzt, dass in den USA jährlich 106.000 Personen an den Folgen einer unerwünschten Arzneimittelwirkung („adverse drug reaction“, ADR) sterben. Das heißt, 0,19\% aller hospitalisierten Patienten erleiden eine tödliche Arzneimittelwirkung. Die von einer norwegischen Arbeitsgruppe durchgeführte prospektive Analyse aller in einer internistischen Abteilung aufgenommenen Patienten ergab eine Inzidenz von $0,95 \%$, an einem arzneimittelinduziertem Ereignis zu versterben [5]. Geriatrische Patienten stellen bedingt durch ihre Vulnerabilität eine besondere Risikogruppe für das Auftreten einer ADR dar. Sowohl

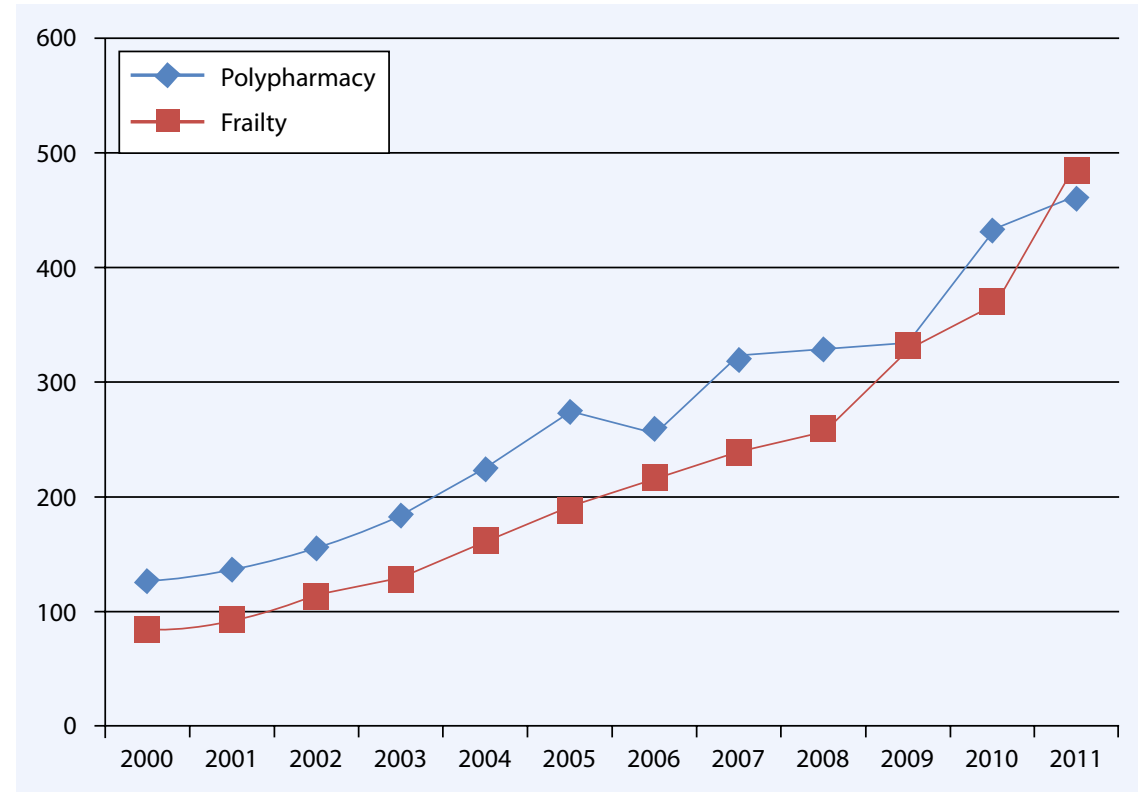

Abb. 1 A In PubMed gelistete Publikationen seit 2000, Suchbegriffe „polypharmacy“ und "frailty" 
hang zeigt deutlich, wie komplex die Fragestellungen in der Pharmakotherapie des geriatrischen Patienten sind. Eine einfache Lösung ist aktuell nicht in Sicht. Leitsätze wie „weniger ist mehr“ führen sehr rasch in ein „undertreatment“. Die Qualität und Sicherheit einer Polypharmazie lassen sich nicht allein an der Anzahl der Substanzen festmachen.

Der aktuelle Themenschwerpunkt schließt sich den vielen wertvollen Initiativen beider Fachgesellschaften nahtlos an. Eine Lösung darf man in Anbetracht der Komplexität nicht erwarten, allerdings sollen die Artikel einen Beitrag zu einer verbesserten und damit auch sicheren Pharmakotherapie leisten. Gassmann et al. weisen in ihrer Arbeit eindrucksvoll auf die Prävalenz der Problematik in einem geriatrischen Patientenkollektiv hin. Zwei weitere Artikel zeigen die möglichen Auswirkungen der Polypharmazie auf eine einzelne Erkrankung - Osteoporose (Gosch et al.) - bzw. auf ein geriatrisches Syndrom - Inkontinenz ( $\mathrm{Ta}$ lasz u. Lechleitner). Einen Überblick über aktuell zur Verfügung stehende Tools im Management der Polypharmazie gibt der Review von Dovjak. Heppner et al. bringen Daten zur Prävalenz und den Folgen von ADR aus der klinischen Praxis. Der Beitrag von Stegemann verdeutlicht, dass das Problem auch für die Pharmaindustrie eine große Herausforderung darstellt.

Wir hoffen, dass der Themenschwerpunkt Ihr Interesse findet und Sie sowohl praktische als auch theoretische Anregungen zum Thema finden.

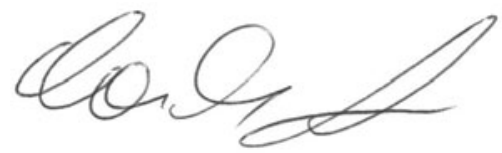

M. Gosch

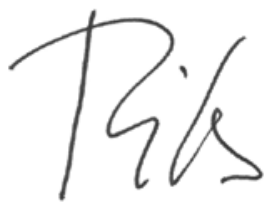

K. Pils

\section{Korrespondenzadresse}

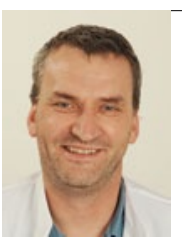

Mag. Dr. M. Gosch

Landeskrankenhaus Hochzirl

Hochzirl 1, 6170 Zirl

Österreich

markus.gosch@tilak.at

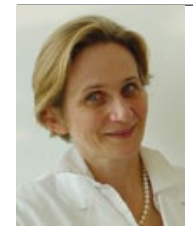

Prim. Dr. K. Pils

Institut für Physikalische

Medizin und Rehabilitation

Sozialmedizinisches Zentrum

Sophienhospital

Apollogasse 19, 1070 Wien

Österreich

Katharina.pils@wienkav.at

\section{Literatur}

1. Jörgensen $T$, Johansson $S$, Kennerfalk $A$, Wallander MA, Svärdsudd K (2001) Prescription drug use, diagnoses, and healthcare utilization among the elderly. Ann Pharmacother 35(9):1004-1009

2. Rottlaender $D$, Scherner $M$, Schneider T, Erdmann E (2007) [Polypharmacy, compliance and non-prescription medication in patients with cardiovascular disease in Germany]. Dtsch Med Wochenschr 132(4):139-144

3. Stoehr GP, Ganguli M, Seaberg EC, Echement DA, Belle $S$ (1997) Over-the-counter medication use in an older rural community: the MoVIES Project. J Am Geriatr Soc 45(2):158-165

4. Lazarou J, Pomeranz BH, Corey PN (1998) Incidence of adverse drug reactions in hospitalized patients - a metaanalysis of prospective studies. JAMA 279:1200-1205

5. Ebbesen J et al. (2001) Drug-related deaths in department of internal medicine. Arch Intern Med 161:2317-2323

6. Deutsche Gesellschaft für Geriatrie. http://www. dggeriatrie.de/dgg/arbeitsgruppen.html. Zugegriffen: 16.05 .2012

7. Österreichische Gesellschaft für Geriatrie und Gerontologie. http://www.geriatrie-online.at/dynasite.cfm?dsmid=110510. Zugegriffen: 16.05 .2012

8. Laroche ML, Charmes JP, Nouaille Y, Picard N, Merle L (2007) Is inappropriate medication use a major cause of adverse drug reactions in the elderly? $\mathrm{Br} \mathrm{J}$ Clin Pharmacol 63(2):177-86

9. Mann E, Böhmdorfer B, Frühwald T (2012) Potentially inappropriate medication in geriatric patients: the Austrian consensus panel list. Wien Klin Wochenschr 124(5-6):160-169

10. Holt S, Schmiedl S, Thürmann PA (2010) Potentially inappropriate medications in the elderly: the PRISCUS list. Dtsch Arztebl Int 107(31-32):543-551

11. Spinewine A, Schmader KE, Barber N et al (2007) Appropriate prescribing in elderly people: how well can it be measured and optimised? Lancet 370(9582):173-184

12. Kuijpers MA, van Marum RJ, Egberts $A C$, Jansen $P A$; OLDY (OLd people Drugs \& dYsregulations) Study Group (2008) Relationship between polypharmacy and underprescribing. Br $\mathrm{I}$ Clin Pharmacol 65(1):130-133 